\title{
Influência de substratos sobre propagação de cambará por meio de estacas ${ }^{1}$
}

\author{
Manoel Ricardo de Andrade Júnior ${ }^{2}$, Mailson Monteiro do Rêgo ${ }^{2}$, Angela Maria dos Santos Pessoa ${ }^{2}$, \\ Bruna Regina dos Santos Silva ${ }^{2}$, Priscila Duarte Silva ${ }^{3}$, Elizanilda Ramalho do Rêgo ${ }^{2}$
}

Resumo: O cambará (Lantana camara L.) pode ser propagado tanto por sementes como por estaquia. Na estaquia um dos principais fatores que afetam o enraizamento é o tipo de substrato. Assim, o objetivo deste trabalho foi avaliar a influência de diferentes substratos sobre propagação de cambará por meio de estacas. Para tanto foram utilizados os seguintes substratos: areia lavada (T1); Plantmax ${ }^{\circledR}$ (T2); vermiculita (T3); pó de coco (T4) e solo (T5). Os dados foram submetidos à análise de variância e as médias comparadas pelo teste de ScottKnott a 5\% de probabilidade. Quando comparou-se as médias verificou-se que a areia lavada e a vermiculita proporcionaram resultados superiores para todas as variáveis analisadas, tendo a areia lavada obtido médias superiores aos demais tratamentos. Para a produção de mudas de cambará recomenda-se o uso de areia lavada e vermiculita como substrato alternativo.

Palavras-chave: estaquia, Lantana camara; substrato.

\section{Influence of substrates on cambará cuttings propagation}

\begin{abstract}
The cambará (Lantana camara L.) can be either propagated by seeds or cuttings. In the cutting propagation method, one of the main factors that affect rooting is the type of substrate. Thus, the objective of this study was to evaluate the influence of different substrates on cambara propagation by cuttings. The following substrates were used: washed sand (T1); Plantmax ${ }^{\circledR}$ (T2); vermiculite (T3); coconut powder (T4) and soil (T5). Data were subjected to analysis of variance and means compared by Scott-Knott test at $5 \%$ probability. It was observed that the washed sand and vermiculite provided superior results for all variables analyzed, and the washed sand promoted higher means than the other treatments. The use of washed sand and vermiculite is, therefore, recommended for the production of cambará seedlings, as an alternative substrate.
\end{abstract}

Keywords: Cuttings; Lantana camara; Substrate.

\footnotetext{
'Submetido em 16/11/2018 e aprovado em 26/08/2019;

${ }^{2}$ Programa de Pós-Graduação em Agronomia, Universidade Federal da Paraíba; Areia, Paraíba, Brasil; E-mail: manoelricardojunior@gmail.com - ORCID: 0000-0002-2074-7377; mailson@cca.ufpb.br - ORCID: 0000-0003-3096-6992; angelapessoapb@gmail.com - ORCID: 0000-0002-7393-984X; bruna-reggina@hotmail.com - ORCID: 0000-0001-7957-3386; elizanilda@cca.ufpb.br - ORCID: 0000-0001-7376-7569;
}

${ }^{3}$ Centro de Ciências Agrárias, Universidade Federal da Paraíba, Areia, Paraíba, Brasil, E-mail: silvapriscilad@gmail.com - ORCID: 00000002-6564-8428. 


\section{Introdução}

O Brasil é reconhecido mundialmente pela riqueza de sua biodiversidade, em que se destacam as plantas nativas para uso ornamental e medicinal como é o caso de algumas espécies da família Verbenaceae. São 16 gêneros e 290 espécies encontradas no país, sendo o maior detentor dessa diversidade. Dentre as espécies, Lantana camara L., também conhecida como lantana, cambará ou cipó-de-cheiro (Howard, 1969, Spies, 1984, Sharma et al., 1988; Salimena e Múlgura, 2014), apresenta potencial como planta ornamental, em função da diversidade de cores de suas flores, dentre elas, citam-se: amarela, branca, laranja e rósea (Lorenzi e Souza, 2008; Passos et al., 2009).

Além de seu uso como planta ornamental, alguns autores também relatam seu uso medicinal, como no tratamento de vermes intestinais e problemas de fertilidade masculina (Baba et al., 2011; Dubey et al., 2011; Kalita et al., 2012; Reddy, 2013; Barros et al., 2016). Além disso, estudos tem comprovado sua eficiência como biopesticidas, no controle de pulgões e outros insetos (Muzemu et al., 2011, Rajasherkar et al., 2014; Mvumi e Maunga, 2018).

No território brasileiro, o cambará encontrase distribuído por todos os estados. Tendo em vista a sua fácil adaptação a regiões tropicais e subtropicais. Classificado como um arbusto, de caule ramificado, formando muitos galhos entrelaçados, podendo apresentar acúleos em algumas espécies (Watanabe, 2016).

Quanto ao tipo de reprodução, essa espécie pode propagar-se tanto por sementes quanto por estacas (Lorenzi e Souza, 2008). A propagação vegetativa especificadamente, é muito importante para a produção de materiais vegetativos clonais em um curto espaço de tempo. Nesse contexto, a estaquia pode ser uma técnica de propagação rápida e de fácil execução, já que é comumente utilizada em espécies que apresentam facilidade para a formação de raízes adventícias (Oliveira et al., 2011). Outro fator de extrema importância para eficiência na propagação vegetativa das plantas é o tipo de substrato utilizado (Finger et al., 2012)

$O$ substrato influência diretamente na formação e arranjo do sistema radicular, e consequentemente, no estado nutricional da planta (Arruda et al., 2007). A escolha do substrato adequado deve levar em consideração uma composição de matéria prima de baixo custo e boas propriedades químicas e físicas que auxiliem no desenvolvimento das raízes adventícias (Costa et al., 2017). O substrato ideal deve permitir uma boa retenção de água, circulação de ar dentro dos espaços porosos, agregação do sistema radicular, uma boa capacidade de troca catiônica, ausência de agentes patogênicos, $\mathrm{pH}$ próximo da neutralidade e baixa salinidade (Farias et al., 2012; Wendling e Gatto, 2012). Embora possua importância ornamental e medicinal, pouco são os trabalhos sobre métodos de propagação vegetativa do cambará (Oliveira et al., 2011).

Diante do exposto, objetivou-se avaliar a influência de diferentes substratos sobre propagação de cambará por meio de estacas.

\section{Material e Métodos}

$\mathrm{O}$ experimento foi realizado em casa de vegetação nas dependências do Laboratório de Biotecnologia e Melhoramento Vegetal do Centro de Ciências Agrárias da Universidade Federal da Paraíba (CCA-UFPB), Areia - PB. O material vegetal utilizado foi proveniente de estacas herbáceas de cambará (L. camara L.) com $20 \mathrm{~cm}$ de comprimento, derivadas de plantas matrizes coletadas no CCA-UFPB.

Para avaliar a eficiência dos diferentes substratos sobre a propagação do cambará foram utilizados os tratamentos: areia lavada (T1); substrato comercial Plantmax ${ }^{\circledR}$ (T2); vermiculita (T3); pó de coco (T4) e solo (T5). Para a caracterização química do substrato (solo) foram realizadas análises químicas do solo (Tabela 1).

Tabela 1 Características químicas do solo utilizados no experimento

\begin{tabular}{|c|c|c|c|c|c|c|c|c|c|c|}
\hline \multirow{2}{*}{$\begin{array}{c}\mathrm{pH}-\mathrm{H}_{2} 0 \\
(1: 2,5)\end{array}$} & $\mathrm{H}^{+}+\mathrm{Al}^{3+}$ & $\mathrm{Al}^{3+}$ & $\mathrm{Ca}^{2+}$ & $\mathrm{Mg}^{2+}$ & $\mathrm{Na}^{+}$ & SB & CTC & \multirow{2}{*}{\multicolumn{2}{|c|}{$\begin{array}{lr} & \mathrm{K}^{+} \\
\mathrm{mg} / \mathrm{dm}^{3}\end{array}$}} & \multirow{3}{*}{$\begin{array}{c}\text { M.O. } \\
\mathrm{g} / \mathrm{kg} \\
50,82\end{array}$} \\
\hline & \multicolumn{7}{|c|}{$\ldots$} & & & \\
\hline 6,4 & 2,61 & 0,00 & 6,98 & 1,40 & 0.07 & 8,73 & 11,34 & 38,27 & 110,70 & \\
\hline
\end{tabular}

CTC - Capacidade de troca de cátions 
Estacas com $20 \mathrm{~cm}$ de comprimento foram postas para enraizar em tubetes de polietileno, com capacidade de $280 \mathrm{~cm}^{3}$, contento os tratamentos. Para manter a umidade no solo, regas diárias foram realizadas.

O delineamento utilizado foi o inteiramente casualizado, com cinco tratamentos e 10 repetições para cada tratamento. A parcela foi constituída por uma estaca por tubete. As avaliações foram realizadas após 30 dias do plantio e verificou-se o aparecimento ou não de calos, raízes e brotações. As variáveis analisadas foram: sobrevivência $=\mathrm{SB}$, número de brotos por estaca $=\mathrm{NB}$, número de folhas $=\mathrm{NF}$, comprimento da maior folha $=\mathrm{CF}(\mathrm{cm})$ e largura da maior folha $=\mathrm{LF}(\mathrm{cm})$. As avaliações foram feitas 30 dias, conforme realizado em outros trabalhos (Loss et al., 2009).

As medidas referentes às dimensões foram tomadas utilizando-se régua graduada transparente, e os valores referentes à quantidade, como o número de estacas sobreviventes foram aferidos por contagem. Todos os dados foram transformados por $\sqrt{0,5}+$ $\mathrm{x}$, submetidos à análise de variância e as médias comparadas pelo teste de Scott-Knott a 5\% de probabilidade. Todas as análises foram realizadas utilizando o programa computacional Genes (Cruz, 2016).

\section{Resultados e Discussão}

As análises de variâncias indicaram efeito significativo pelo teste $\mathrm{F}(\mathrm{p}<0,05)$ entre os tratamentos para todas as características avaliadas (Tabela 2), sobrevivencia(SB), número de brotos (NB), número de folhas (NF), comprimento da folha (CF) e largura das folhas (LF). Esse resultado indica que os substratos influenciaram positivamente no desempenho dessas características.

$\mathrm{O}$ coeficiente de variação $(\mathrm{CV})$ variou entre $25,09 \%$ a $68,62 \%$ para as características avaliadas (Tabela 2). Apesar da alta variação, os valores são aceitáveis pelo fato de que cada variável possui uma escala particular de coeficiente de variação, sendo relacionada à sua variabilidade intrínseca (Zimmermann, 2014). Crispim et al. (2015) avaliando diferentes substratos no enraizamento de estacas de Pyrostegia venusta, planta ornamental, também verificaram oscilações elevadas no $\mathrm{CV}$ em relação a diferentes variáveis analisadas.

Tabela 2 Resumo da análise de variância (quadrados médios) para cinco características relacionadas à propagação por estaquia de Cambará (Lantana camara L.)

\begin{tabular}{lllllll}
\hline \multirow{2}{*}{ Fontes de variação } & \multirow{2}{*}{ GL } & \multicolumn{5}{c}{ Quadrados médios } \\
& & SB & NB & NF & CF & LF \\
\hline Tratamentos & 4 & $0,17^{*}$ & $0,59^{* *}$ & $3,34^{*}$ & $0,56^{*}$ & $0,40^{*}$ \\
Resíduo & 45 & 0,04 & 0,15 & 0,94 & 0,21 & 0,15 \\
\hline CV $(\%)$ & - & 25,09 & 39,16 & 68,62 & 45,65 & 4,37
\end{tabular}

*Significativo em nível de 5\% de probabilidade pelo teste F. SB - Sobrevivência; NB - Número de brotos; Número de folhas; CF - Comprimento da folha e LF - Largura da folha.

Na comparação das médias pelo teste de Scott-Knott a $5 \%$ de probabilidade, verificou-se que a areia lavada e a vermiculita proporcionaram resultados superiores para todas as variáveis analisadas (Tabela 3). Uma das características ideias dos substratos para o enraizamento das estacas é proporcionar umidade e aeração (Ming et al., 1998), tanto a areia quanto a vermiculita apresenta essas características. Gomes et al. (2015) relataram que substratos ideais para produção de mudas devem possuir baixa densidade, boa capacidade de absorção e retenção de água, boa aeração e drenagem. Tanto a areia quanto a vermiculita apresenta essas características.

Os resultados observados para o substrato comercial $\left(\right.$ Plantmax $^{\circledR}$ ), pó de colo e solo quando comparados com os observados em areia e vermiculita, indicam que o enraizamento de estacas dessa espécie é favorecido em substratos que apresentem drenagem rápida e baixo poder de retenção de água. Assim, de acordo com Garay et al. (2014), devido ao fato de que cada 
substrato possuirá suas vantagens e desvantagens, não há um substrato ideal, contudo, é possível escolher um bom material, os resultados dependerão também das características da cultura, sendo dessa forma imprescindível testar diferentes substratos ou misturas de substratos para cada espécie.

Os resultados encontrados nesse trabalho, provavelmente demonstram que $L$. camara $\mathrm{L}$. é uma espécie mais exigente em aeração e menos umidade. O substrato areia e vermiculita possui maior quantidade de macroporos, facilitando a circulação de ar entre as partículas e a consequente aeração na base da estaca, diminuindo a mortalidade das mesmas e facilitando o enraizamento. Além disso, possivelmente o cambará pode ser intolerante a substratos ou ambientes encharcados, já que a areia possui baixa capacidade de retenção de água (Wendling et al., 2002), permitindo trocas gasosas eficientes, pois quanto maior a disponibilidade de oxigênio na base das estacas maior será a eficiência da atividade celular durante $\mathrm{o}$ processo de formação de calos e emissão de raízes (Hartmann et al. 2002), favorecendo dessa forma o enraizamento.

Tabela 3 Comparação de médias do tipo de substrato sobre caracteres relacionados à propagação por estaquia de Cambará (Lantana camara L.)

\begin{tabular}{cccccc}
\hline \multirow{2}{*}{ Tratamentos } & \multicolumn{5}{c}{ Características } \\
& SB (\%) & NB & NF & CF $(\mathrm{cm})$ & LF $(\mathrm{cm})$ \\
\hline Areia lavada (T1) & $1,06 \mathrm{a}$ & $1,36 \mathrm{a}$ & $2,21 \mathrm{a}$ & $1,32 \mathrm{a}$ & $1,21 \mathrm{a}$ \\
Plantmax $^{\circledR}$ (T2) & $0,86 \mathrm{~b}$ & $0,93 \mathrm{~b}$ & $1,18 \mathrm{~b}$ & $0,90 \mathrm{~b}$ & $0,88 \mathrm{~b}$ \\
Vermiculita (T3) & $0,96 \mathrm{a}$ & $1,13 \mathrm{a}$ & $1,82 \mathrm{a}$ & $1,20 \mathrm{a}$ & $1,12 \mathrm{a}$ \\
Pó de coco (T4) & $0,76 \mathrm{~b}$ & $0,79 \mathrm{~b}$ & $0,89 \mathrm{~b}$ & $0,81 \mathrm{~b}$ & $0,78 \mathrm{~b}$ \\
Solo (T5) & $0,76 \mathrm{~b}$ & $0,79 \mathrm{~b}$ & $0,96 \mathrm{~b}$ & $0,81 \mathrm{~b}$ & $0,79 \mathrm{~b}$ \\
\hline
\end{tabular}

*Médias seguidas pela mesma letra nas colunas não diferem significativamente entre si pelo teste de Scott-Knott ao nível de $5 \%$ de probabilidade. SB - Sobrevivência; NB - Número de brotos; NF - Número de folhas; CF - Comprimento da folha e Largura da folha.

\section{Conclusão}

Para a produção de mudas de cambará recomenda-se o uso de areia lavada e vermiculita como substrato alternativo.

\section{Referências}

Arruda, M. R.; Pereira, J. C. R.; Moreira, A.; Teixeira, W. G. Enraizamento de estacas herváceas de guaranazeiro em diferentes substratos. In Embrapa Pecuária SudesteArtigo em anais de congresso (ALICE). Ciência e Agrotecnologia, v. 31, n. 1, p. 236241, 2007., 2007. http://www.scielo.br/pdf/\%0D/cagro/v31n1/v31n1a35.pdf

Baba, G.; Adewumi ,A. A. J.; Aina, V. O.; Phytochemical Characterization and invivo Anti-Malaria Activity of Lantana camara Leaf Extract. British Journal of Pharmacology and Toxicology, v. 2, n. 6, p. 277-282, 2011.
Barros, L. M.; Duarte, A. E.; Morais-Braga, M. F. B.; Waczuk, E. P.; Vega, C.; Leite, N. F.; Menezes, I. R. A. de.; Coutinho, H. D. M.; Kamdem, J. P. Chemical characterization and trypanocidal, leishmanicidal and cytotoxicity potential of Lantana camara L. (Verbenaceae) essential oil. Molecules, v. 21, n. 2, p. 2-9, 2016. https://doi.org/10.3390/molecules21020209

Costa, A. C. M.; Alves Chiba, H. S. Characterization of the production practices used by flower and ornamental plants producers in Santarém-PA (Brasil). Revista Espacios, v. 38, n. 38, p. 21, 2017. https://www.revistaespacios.com/a17v38n28/17382821.ht $\mathrm{ml}$

Crispim, J. G.; Rêgo, M. M.; Rêgo, E. R.; Pessoa, A. M. S; Barroso, P. A. Utilização de diferentes substratos na propagação de Pyrostegia venusta através de estacas. Revista Verde de Agroecologia e Desenvolvimento 
Sustentável, v. 10, n. 4, p.38-41, 2015. https://doi.org/10.18378/rvads.v10i4.3375

Cruz, C. D. Genes Software-extended and integrated with the $\mathrm{R}$, Matlab and Selegen. Acta Scientiarum. Agronomy, v. 38, n. $4, \quad$ p. 547-552, 2016. http://dx.doi.org/10.4025/actasciagron.v38i4.32629

Dubey, M.; Sharma, S.; Sengar, S.; Bhadauria, R. K. S. Gautam in vitro antibacterial activity of Lantana camara leaves hydrosol. Journal of Pharmacy Research, v. 4, n. 11, p. 39723974 , 2011. http://citeseerx.ist.psu.edu/viewdoc/download?doi=10.1.1 $.736 .252 \&$ rep $=$ rep $1 \&$ type $=$ pdf

Farias, W. C.; Oliveira, L. L. P.; Oliveira, T. A.; Dantas, L. L. G. R. D.; Silva, T. A. G. Caracterização física de substratos alternativos para a produção de mudas. Agropecuária Científica no Semiárido, v. 8, n. 3, p. 1-6, 2012.

http://revistas.ufcg.edu.br/acsa/index.php/ACSA/article/vi ew/187

Finger, F. L.; Rêgo, E. R.; Segatto, F. B.; Nascimento, N. F. F.; Rêgo, M. M. Produção e potencial de mercado para pimenta ornamental. Informe Agropecuário, v. 33, n. 267, p. 14 20, 2012.

Garay, C. R. E.; Bogarin, N. B. G.; Oviedo, V. R. S. Producción de mudas de tomate en el sistema flotante. Investigación Agraria, v. 16, n. $2, \quad$ p. 129-135, 2014. http://www.agr.una.py/revista/index.php/ria/article/view/ 252

Gomes, J. A. O.; Teixeira, D. A.; Marques, A. P. S.; Bonfim, F. P. G. Diferentes substratos na propagação por estaquia de assa-peixe (Vernonia polyanthes Less). Revista Brasileira de Plantas Medicinais, v. 17, n. 4, p.1177-1181, 2015. http://dx.doi.org/10.1590/1983084x/15_008

Hartmann, H.T.; Kester, D.E.; Davies Júnior, R.T.; Geneve, R.L. Plant propagation: principles e practices. $7^{\text {th }}$ ed. New Jersey: Prentice Hall, 2002. 880p.

Howard, R. A. A check list of cultivar names used in the genus Lantana. Arnoldia, v. 29, p. 73-109, 1969.

Kalita, S.; Kumar, G.; Karthik, L.; Rao, K. V. B. A review on medicinal properties of Lantana camara Linn. Research Journal of Pharmacy and Technology, v. 5, n. 6, p. 711-715, 2012. https://pdfs.semanticscholar.org/7cb2/a0cbbf562a1e8a35 99a3a38c6cf8b8de3bd8.pdf

Loss, A., Teixeira, M. B., Santos, T. D. J., Gomes, V. M., \& Queiroz, L. H. (2009). Indução do enraizamento em estacas de Malvaviscus arboreus Cav. com diferentes concentrações de ácido indol-butírico (AIB). Acta Scientiarum. Agronomy, v. 31, n. 2, p. 269-273, 2009. http://dx.doi.org/10.1590/S180786212009000200013

Lorenzi, H.; Souza, H. M. de. Plantas ornamentais no Brasil: arbustivas, herbáceas e trepadeiras. 4. ed. Nova Odessa, Sp: Instituto Plantaram de Estudos da Flora Ltda, 2008.

Ming, L. C., Scheffer, M. C., Júnior, C., Barros, I. B. I., Mattos, J. . Plantas madicinais, aromáticas e codimentares: avanços na pesquisa agronômica. v.2, apoio PROIN/CAPES. Botucatu: UNESP, 1998. 238

Muzemu, S.; Mvumi, B.M.; Nyirenda, S. O. M.; Sileshi, G. W.; Sola, P.; Chikukura, I.; Kamunula, J.F.; Belmain, S. R.; Stevenson, P. C. Pesticidal effects of indigenous plant extracts against rape aphids and tomato red spider mites. African Crop Science Conference Proceedings, v. 10, p. 169-171, $2011 . \quad$ http://outputs.worldagroforestry.org/cgibin/koha/opac-detail.pl?biblionumber $=34779$

Mvumi, C.; Maunga, P. R. Efficacy of lantana (Lantana camara) extract application against aphids (Brevicoryne brassicae) in rape (Brassica napus) over varied periods of time. African Journal of Biotechnology, v. 17, n. 8, p. 249-254, 2018. https://doi.org/10.5897/ajb2017.16160

Oliveira, A. C. L.; Arrigoni-Blank, M. F.; Blank, A. F.; Bianchini, F. G Produção de mudas de dois genótipos de alecrim-de-tabuleiro (Lippia gracilis Schauer) em função de fertilizante mineral, calcário, substratos e recipientes. Revista Brasileira de Plantas Medicinais, v. 13, n. 1, p. 35-42, 2011. http://dx.doi.org/10.1590/S1516-05722011000100006

Passos, J. L.; Meira, R. M. S. A.; Barbosa, L. C. A. Foliar anatomy of the species Lantana camara and L. radula (Verbenaceae). Planta Daninha, v. 27, n. 4, p. 689-700, 2009. http://dx.doi.org/10.1590/S0100-83582009000400007

Rajashekar, Y.; Ravindra, K. V.; Bakthavatsalam, N. Leaves of Lantana camara Linn. (Verbenaceae) as a potential insecticide 
for the management of three species of stored grain insect pests. Journal of food science and technology. v. 51, n. 11, p. 3494-3499, 2014. https://doi.org/10.1007/s13197-012-0884-8

Reddy, N. M. Lantana camara Linn. chemical constituents and medicinal properties: a review. Scholars Academic Journal of Pharmacy, v. 2, n. 6, p. 445-448, 2013. https://pdfs.semanticscholar.org/6033/f32b97b42dbcf8ad 1a242ff8efb7b6c06bff.pdf

Sharma, O. P.; Makkar, H. P. S.; Dawra, R. K.; A review of the noxious plant Lantana camara. Toxicon, v.26, n.11, p. 975-987, 1988. https://doi.org/10.1016/0041-0101(88)90196-1

Salimena, F. R.; Múlgura, M. E. Notas taxonômicas em Verbenaceae J. St. Hil. do Brasil. Rodriguésia-Instituto de Pesquisas Jardim Botânico do Rio de Janeiro, v. 66, n. 1, p. 191-197, 2014. http://rodriguesiaseer.jbrj.gov.br/index.php/rodriguesia/article/view/987/0

Souza, F. X. Materiais para formulação de substratos na produção de mudas e no cultivo de plantas envasadas. Embrapa Agroindústria Tropical-Documentos (INFOTECA-E), 2001. https://www.infoteca.cnptia.embrapa.br/bitstream/doc/42 3625/1/Dc043.pdf

Spies, J. J. A cytotaxonomic study of Lantana camara (Verbenaceae) from South Africa. South African Journal of Botany, v. 3, n. 4, p. 231-250, 1984. https://doi.org/10.1016/S00224618(16)30034-1

Wendling, I.; Gatto, A. Substratos para produção de mudas: Características essenciais de um substrato. In: Wendling, Ivar; Souza, Hermes Moreira de. Substratos, adubação e irrigação na produção de mudas. $2^{\mathrm{a}}$ ed. Viçosa: Aprenda Fácil, 2012. Cap. 1. p. 15-16.

Wendling, I.; Gatto, A.; Paiva, H.N.; Gonçalves, W. Substratos, adubação e irrigação na produção de mudas. $1^{\mathrm{a}}$ ed. Viçosa: Aprenda Fácil Editora, p. 165, 2002.

Watanabe, M. A. Ácaros em Lantana camara. Embrapa Meio Ambiente, Informativo n. 51, 2005.

Barros, L. M.; Duarte, A. E.; Morais-Braga, M. F. B.; Waczuk, E. P.; Veja, C.; Leite, N. F.; Menezes, I. R. A. de; Coutinho, H. D. M.; Rocha, J. B. T.; Kamdem, J. P. Chemical characterization and trypanocidal, leishmanicidal and cytotoxicity potential of Lantana camara L. (Verbenaceae) essential oil. Molecules, v. 21, n. 2, p. 209, 2016. https://doi.org/10.3390/molecules21020209

Zimmermann, F. J. P. Conceitos gerais e definições úteis.: Medidas de dispersão. In: Zimmermann, F. J. P. Estatística aplicada à pesquisa agrícola. $2^{\mathrm{a}}$ ed. Brasília: Embrapa, 2014. Cap. 1, p. 28. 